\title{
A skein relation for the HOMFLYPT polynomials of two-cable links
}

\author{
TAIZO KANENOBU
}

\begin{abstract}
We give a skein relation for the HOMFLYPT polynomials of 2-cable links. We have constructed arbitrarily many 2-bridge knots sharing the same HOMFLYPT, Kauffman, and Links-Gould polynomials, and arbitrarily many 2-bridge links sharing the same HOMFLYPT, Kauffman, Links-Gould, and 2-variable Alexander polynomials. Using the skein relation, we show their 2-cable links also share the same HOMFLYPT polynomials.
\end{abstract}

57M25, 57M27

\section{Introduction}

Soon after the discovery of the HOMFLYPT polynomials (Freyd et al [5] and Przytycki and Traczyk [24]), Morton and Short [22] and Yamada [28] gave examples of a pair of knots with the same HOMFLYPT polynomial that are distinguished by the HOMFLYPT polynomials of their 2-cable links. Moreover, Przytyzki [23], and Lickorish and Lipson [19] showed that if two knots are mutant knots, their 2-cable links share the same HOMFLYPT polynomial; this is false if they are links of more than one component. Recently, Stoimenow [26] found the first examples of four pairs of non-mutant 12crossing knots whose 2-cable links share the same HOMFLYPT polynomials. They are

$$
\left\{12_{341}, 12_{627}\right\}, \quad\left\{12_{1305}, 12_{1872} !\right\}, \quad\left\{12_{1378}, 12_{1704}\right\}, \quad\left\{12_{1423}, 12_{1704}\right\}
$$

from the table of Hoste, Thistlethwaite, and Weeks [6], where $12_{1872}$ ! is the mirror image of $12_{1872}$ and $\left\{12_{1378}, 12_{1423}\right\}$ is a mutant pair.

On the other hand, in 1992, JR Links and MD Gould [21] discovered a 2-variable polynomial invariant for an oriented link, which we call the LG polynomial. It is known that mutant links share the same LG polynomial (De Wit-Links-Kauffman [4]) and all prime knots with less than or equal to 10 crossings are completely classified by the LG polynomial (De Wit [2]). By using skein relations given by De Wit et al [4] and Ishii [7], Ishii and the author [8] gave several examples of different knots and links sharing 
the same LG polynomials; the smallest such example is a pair of 2-bridge knots with 14 crossings; see Example 5.4. Then De Wit and Links [3] searched for non-mutant pair of prime knots with 11 and 12 crossings sharing the same LG polynomial, and they discovered such pairs. Surprisingly, they agree with the above knot pairs (1) found by Stoimenow, that is, the four pairs (1) are the smallest ones of non-mutant knots whose 2-cable links share the same HOMFLYPT polynomials. This suggests some relation between the HOMFLYPT polynomial of a 2-cable link and the LG polynomial. This motivated the author to discover a skein relation for the HOMFLYPT polynomial of 2-cable links (Theorem 2.1, Corollary 3.3 and Corollary 3.4), which is similar to one for the LG polynomial.

In [8], we have constructed examples of arbitrarily many 2-bridge knots or links sharing the same LG polynomial as well as the HOMFLYPT and Kauffman polynomials [17; 18]; cf Kanenobu [10; 11; 12; 13; 14] and Kanenobu and Sumi [15; 16]. Our skein relation enables us to prove that their 2-cable links share the same HOMFLYPT polynomials (Theorem 5.1 and Theorem 5.2). Also, we can construct some other examples with the same 2-cable HOMFLYPT polynomials, which are special case considered in Ishii and Kanenobu [8].

This paper consists of five sections. In Section 2, we prove the skein relation for the 2-cable HOMFLYPT polynomial (Theorem 2.1). In Section 3, we define the 2-cable links, give some properties on the HOMFLYPT polynomial (Proposition 3.1 and Proposition 3.2), and give two corollaries for Theorem 2.1 (Corollary 3.3 and Corollary 3.4). In Section 4, we consider the 2-cable HOMFLYPT polynomials of the link $K\left[\beta ; R_{1}, R_{2}, \ldots, R_{n}\right]$, which had been given in [8]. In Section 5, we prove the above-mentioned Theorem 5.1 and Theorem 5.2.

\section{Skein relation}

The HOMFLYPT polynomial $P(L ; v, z) \in \boldsymbol{Z}\left[v^{ \pm 1}, z^{ \pm 1}\right]$ (Freyd et al [5], Jones [9] and Przytycki and Traczyk [24]) is an invariant of the isotopy type of an oriented link $L$, which is defined by the following formulas:

$$
\begin{gathered}
P(U ; v, z)=1 ; \\
v^{-1} P\left(L_{+} ; v, z\right)-v P\left(L_{-} ; v, z\right)=z P\left(L_{0} ; v, z\right) .
\end{gathered}
$$

where $U$ is the unknot and $L_{+}, L_{-}, L_{0}$ are three links that are identical except near one point where they are as in Figure 1. Equation (3) implies the following, which we 
will use often.

$$
\begin{aligned}
& P\left(L_{+} ; v, z\right)=v^{2} P\left(L_{-} ; v, z\right)+v z P\left(L_{0} ; v, z\right), \\
& P\left(L_{-} ; v, z\right)=v^{-2} P\left(L_{+} ; v, z\right)-v^{-1} z P\left(L_{0} ; v, z\right) .
\end{aligned}
$$
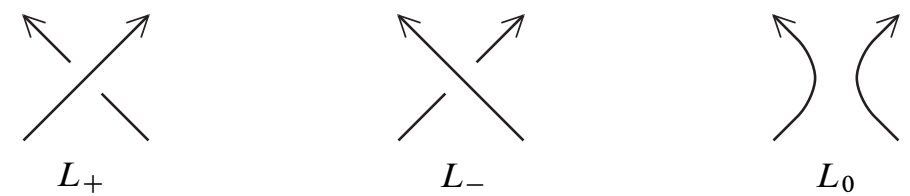

Figure 1

Let $L\left(\boldsymbol{t}_{+}\right), L\left(\boldsymbol{t}_{-}\right), L\left(\boldsymbol{e}_{+}\right), L\left(\boldsymbol{e}_{-}\right), L\left(\boldsymbol{f}_{+}\right), L\left(\boldsymbol{f}_{0}\right), L\left(\boldsymbol{f}_{-}\right)$be oriented links identical outside a ball and inside are 8-end tangles $\boldsymbol{t}_{+}, \boldsymbol{t}_{-}, \boldsymbol{e}_{+}, \boldsymbol{e}_{-}, \boldsymbol{f}_{+}, \boldsymbol{f}_{0}$, $\boldsymbol{f}_{-}$as shown in Figure 2, respectively. We denote the HOMFLYPT polynomial of the link $L(s)$ by $P(s)$, where $s$ is one of these tangles.

\section{Theorem 2.1}

(6)

$$
\begin{aligned}
& v^{-5} P\left(\boldsymbol{t}_{+}\right)+v^{5} P\left(\boldsymbol{t}_{-}\right) \\
= & v^{-3} P\left(\boldsymbol{e}_{+}\right)+v^{3} P\left(\boldsymbol{e}_{-}\right)+\left(v^{-3} P\left(\boldsymbol{f}_{+}\right)+\left(v^{-1}+v\right) P\left(\boldsymbol{f}_{0}\right)+v^{3} P\left(\boldsymbol{f}_{-}\right)\right) z^{2} .
\end{aligned}
$$

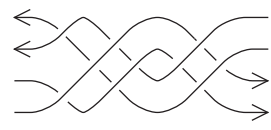

$\boldsymbol{t}_{+}$

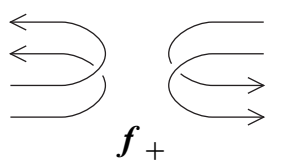

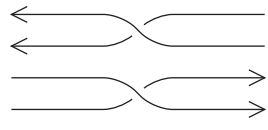

$e_{+}$

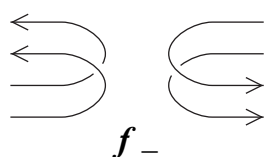

Figure 2

Remark 2.2 We denote the LG polynomial of an oriented link by $\operatorname{LG}(L)$, which is a 2 -variable polynomial in variables $t_{0}$ and $t_{1}$. The formula (6) is similar to the skein 
relation for the LG polynomial [8, Equation (3)]:

$$
\mathrm{LG}\left(L_{+2}\right)+t_{0} t_{1} \mathrm{LG}\left(L_{-2}\right)=\left(t_{0} t_{1}+1\right) \mathrm{LG}\left(L_{0}\right)-2\left(t_{0}-1\right)\left(t_{1}-1\right) \mathrm{LG}\left(L_{\infty}\right),
$$

which is obtained from the relations [8, Equations (1) and (2)] given by De Wit et al [4] and Ishii [7], respectively. Here, $L_{+2}, L_{-2}, L_{0}, L_{\infty}$ are four oriented links that are identical except near one point where they are as in Figure 12.

Except for the 8-end tangles given in Figure 2, we use tangles as shown in Figure 3. Note that these tangle diagrams have only positive crossings.
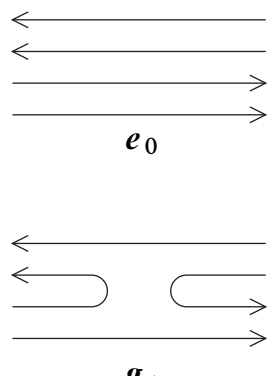

$\boldsymbol{g}_{1}$

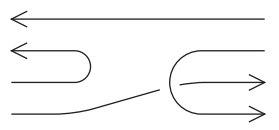

$\boldsymbol{h}_{1}$

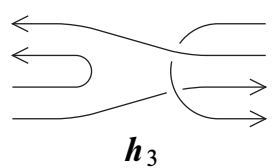

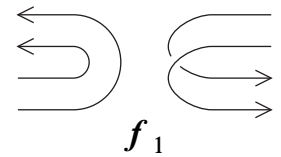

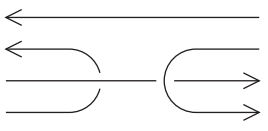

$\boldsymbol{g}_{2}$

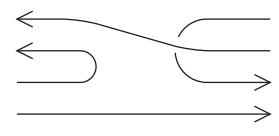

$\boldsymbol{h}_{2}$

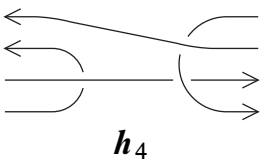

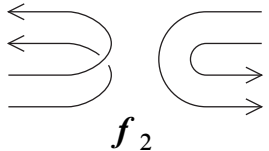

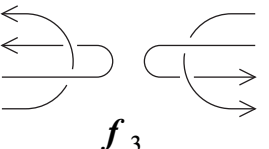

$f_{3}$

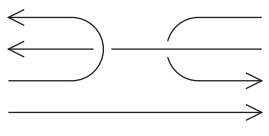

$\boldsymbol{g}_{3}$

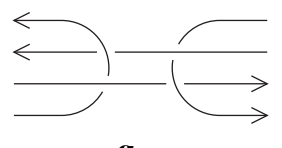

$\boldsymbol{g}_{4}$

Figure 3

Lemma 2.3 Let $\boldsymbol{t}_{1}$ be the 8-end tangle as shown in Figure 4. Then we have:

$$
\begin{aligned}
P\left(\boldsymbol{t}_{1}\right)= & v^{8} P\left(\boldsymbol{e}_{0}\right)+\left(v^{7} P\left(\boldsymbol{g}_{1}\right)+v^{5} P\left(\boldsymbol{g}_{2}\right)+v^{5} P\left(\boldsymbol{g}_{3}\right)+v^{3} P\left(\boldsymbol{g}_{4}\right)\right) z \\
& +\left(v^{4} P\left(\boldsymbol{f}_{+}\right)+v^{2} P\left(\boldsymbol{f}_{3}\right)\right) z^{2} .
\end{aligned}
$$




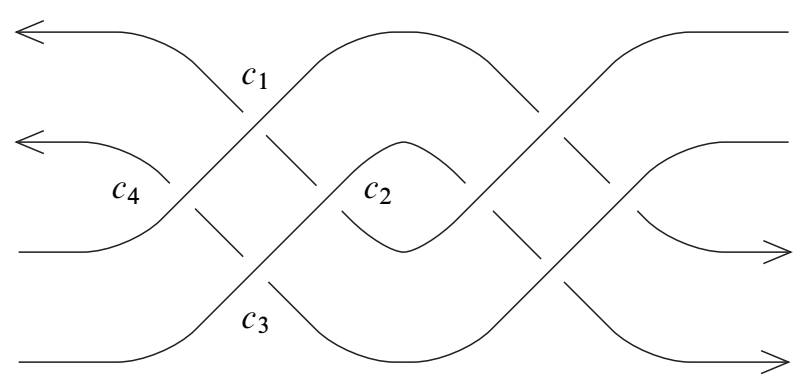

Figure 4

Proof First, we consider the right-hand side of (7). Applying (4) two times, we have

$$
P\left(\boldsymbol{f}_{3}\right)=v^{4} P\left(\boldsymbol{f}_{0}\right)+v^{3} z P\left(\boldsymbol{f}_{1}\right)+v^{3} z P\left(\boldsymbol{f}_{2}\right)+v^{2} z^{2} P\left(\boldsymbol{f}_{+}\right),
$$

Thus the right-hand side of (7) is equal to

(9)

$$
\begin{aligned}
& v^{8} P\left(\boldsymbol{e}_{0}\right)+\left(v^{4} z^{2}+v^{4} z^{4}\right) P\left(\boldsymbol{f}_{+}\right)+v^{6} z^{2} P\left(\boldsymbol{f}_{0}\right)+v^{5} z^{3} P\left(\boldsymbol{f}_{1}\right)+v^{5} z^{3} P\left(\boldsymbol{f}_{2}\right) \\
& +\left(v^{7} P\left(\boldsymbol{g}_{1}\right)+v^{5} P\left(\boldsymbol{g}_{2}\right)+v^{5} P\left(\boldsymbol{g}_{3}\right)+v^{3} P\left(\boldsymbol{g}_{4}\right)\right) z
\end{aligned}
$$

Next, we consider the left-hand side of (7), $P\left(\boldsymbol{t}_{1}\right)$. For $\delta=\left(\delta_{1}, \delta_{2}, \delta_{3}, \delta_{4}\right)$, where each $\delta_{i}$ is either a minus sign or zero, we denote by $\boldsymbol{t}_{\delta}$ the 8-end tangle obtained from $\boldsymbol{t}$ by changing the positive crossing $c_{i}$ to a negative crossing or smoothing according as $\delta_{i}$ is a minus sign or zero. Then applying (4), we have

$$
P\left(\boldsymbol{t}_{1}\right)=\sum_{\delta} v^{2 m_{\delta}}(v z)^{n_{\delta}} P\left(\boldsymbol{t}_{\delta}\right)
$$

where $m_{\delta}$ and $n_{\delta}$ are the numbers of minus signs and zeros in $\delta$, respectively. Applying (4) and (5), we obtain the following:

$$
\begin{aligned}
& P\left(\boldsymbol{t}_{0000}\right)=P(\stackrel{\longleftrightarrow}{\longrightarrow} \underset{\longrightarrow}{\longrightarrow})=v^{2} P\left(\boldsymbol{f}_{0}\right)+v z P\left(\boldsymbol{f}_{1}\right) ; \\
& P\left(\boldsymbol{t}_{-000}\right)=P\left(\boldsymbol{t}_{00-0}\right)=P\left(\boldsymbol{h}_{3}\right) \text {; } \\
& P\left(\boldsymbol{t}_{0-00}\right)=P\left(\boldsymbol{f}_{1}\right) \text {; } \\
& P\left(\boldsymbol{t}_{000-}\right)=P(\stackrel{\longrightarrow}{\longrightarrow} \underset{\longrightarrow}{\longrightarrow})
\end{aligned}
$$




$$
\begin{aligned}
= & v^{-2} P(\stackrel{\longrightarrow}{\longrightarrow}(\underset{\longrightarrow}{\longrightarrow}) \\
& -v^{-1} z P(\stackrel{\longrightarrow}{\longrightarrow}) \\
= & v^{-2}\left(v^{2} P\left(\boldsymbol{f}_{2}\right)+v z P\left(\boldsymbol{f}_{+}\right)\right)-v^{-1} z P\left(v^{2} P\left(\boldsymbol{f}_{0}\right)+v z P\left(\boldsymbol{f}_{1}\right)\right) \\
= & v^{-1} z P\left(\boldsymbol{f}_{+}\right)-v z P\left(\boldsymbol{f}_{0}\right)-z^{2} P\left(\boldsymbol{f}_{1}\right)+P\left(\boldsymbol{f}_{2}\right) ;
\end{aligned}
$$

(15) $P\left(\boldsymbol{t}_{--00}\right)=P\left(\boldsymbol{h}_{1}\right)$;

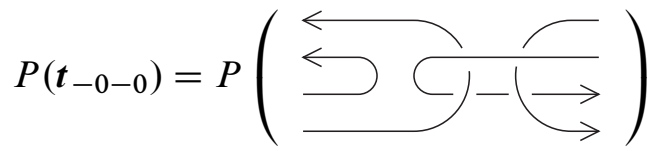

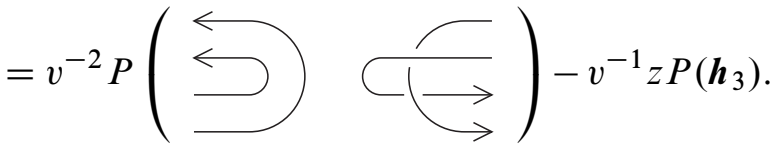

$$
\begin{aligned}
& =v^{-2}\left(v^{2} P\left(\boldsymbol{f}_{0}\right)+v z P\left(\boldsymbol{f}_{1}\right)\right)-v^{-1} z P\left(\boldsymbol{h}_{3}\right) \\
& =P\left(\boldsymbol{f}_{0}\right)+v^{-1} z P\left(\boldsymbol{f}_{1}\right)-v^{-1} z P\left(\boldsymbol{h}_{3}\right) \text {; }
\end{aligned}
$$

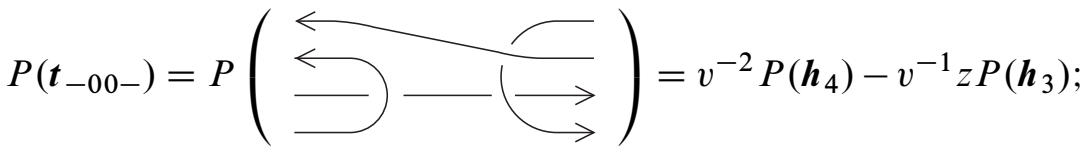

$$
P\left(\boldsymbol{t}_{0--0}\right)=P\left(\boldsymbol{h}_{2}\right) \text {; }
$$

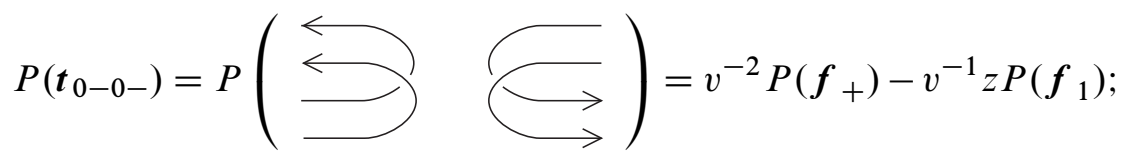

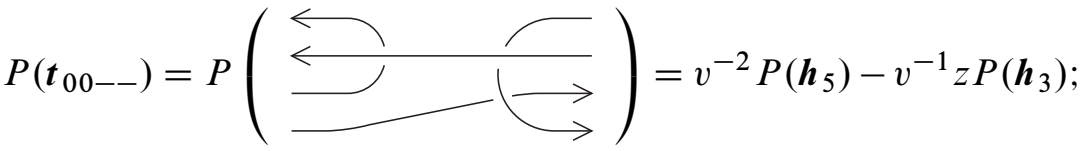

(21) $P\left(\boldsymbol{t}_{---0}\right)=P\left(\boldsymbol{g}_{1}\right)$;

$$
\begin{aligned}
& P\left(\boldsymbol{t}_{--0-}\right)=P(\stackrel{\longleftrightarrow \longrightarrow}{\longrightarrow})=v^{-2} P\left(\boldsymbol{g}_{2}\right)-v^{-1} z P\left(\boldsymbol{h}_{1}\right) ;
\end{aligned}
$$

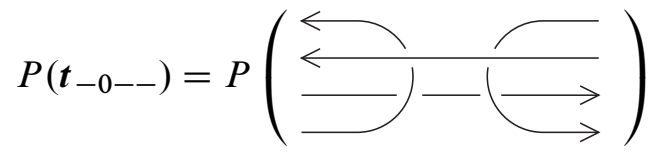




$$
\begin{aligned}
& =v^{-4} P\left(\boldsymbol{g}_{4}\right)-v^{-3} z P\left(\boldsymbol{h}_{4}\right)-v^{-3} z P\left(\boldsymbol{h}_{5}\right)+v^{-2} z^{2} P\left(\boldsymbol{h}_{3}\right) \text {; }
\end{aligned}
$$

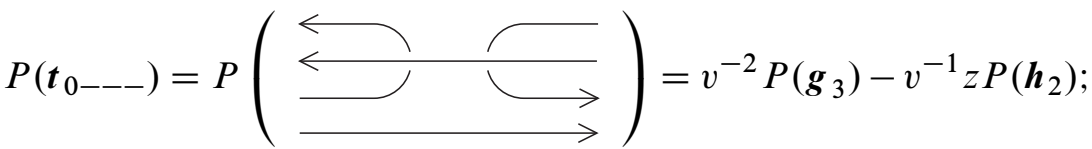

$$
P\left(t_{----}\right)=P\left(e_{0}\right) .
$$

Substituting (11)-(25) into (10), we obtain (9). This completes the proof.

Proof of Theorem 2.1 By adding two negative crossings to an 8-end tangle as in Figure 5(a), $t_{1}, e_{0}, g_{1}, g_{2}, g_{3}, g_{4}, f_{+}, f_{3}$ become $t_{+}, e_{-}, k_{1}, k_{2}, k_{3}$, $\boldsymbol{k}_{4}, \boldsymbol{f}_{0}, \boldsymbol{f}_{+}$, respectively, where $\boldsymbol{k}_{1}, \ldots, \boldsymbol{k}_{4}$ are tangles as shown in Figure 6 . Thus (7) becomes

$$
\begin{array}{rl}
P\left(\boldsymbol{t}_{+}\right)=v^{8} & P\left(\boldsymbol{e}_{-}\right)+\left(v^{7} P\left(\boldsymbol{k}_{1}\right)+v^{5} P\left(\boldsymbol{k}_{2}\right)+v^{5} P\left(\boldsymbol{k}_{3}\right)+v^{3} P\left(\boldsymbol{k}_{4}\right)\right) z \\
+ & \left(v^{4} P\left(\boldsymbol{f}_{0}\right)+v^{2} P\left(\boldsymbol{f}_{+}\right)\right) z^{2}
\end{array}
$$

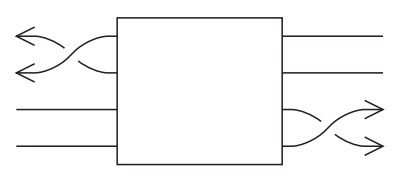

(a)

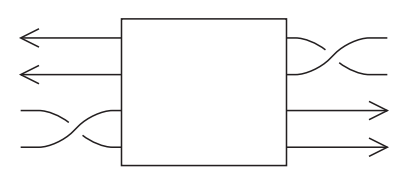

(b)

Figure 5

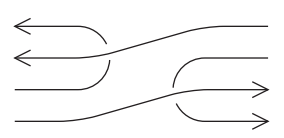

$\boldsymbol{k}_{1}$

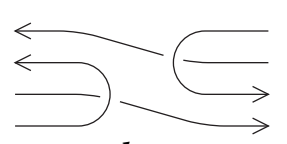

$\boldsymbol{k}_{5}$

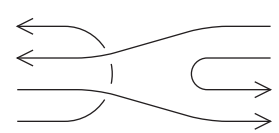

$\boldsymbol{k}_{2}$

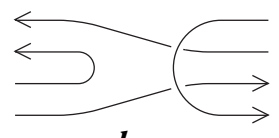

$\boldsymbol{k}_{6}$

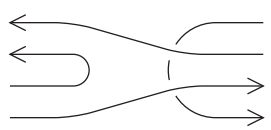

$\boldsymbol{k}_{3}$

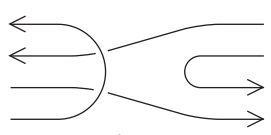

$\boldsymbol{k}_{7}$
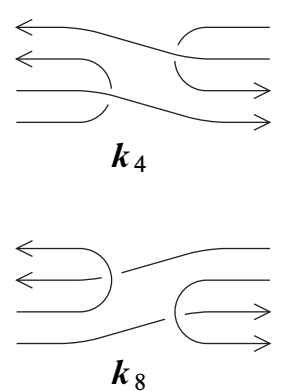

Figure 6

Algebraic $\&$ Geometric $\mathcal{T}$ opology, Volume 7 (2007) 
Similarly, adding two negative crossings to an 8-end tangle as in Figure 5(b), we obtain

$$
\begin{aligned}
P\left(\boldsymbol{t}_{+}\right)= & v^{8} P\left(\boldsymbol{e}_{-}\right)+\left(v^{7} P\left(\boldsymbol{k}_{5}\right)+v^{5} P\left(\boldsymbol{k}_{6}\right)+v^{5} P\left(\boldsymbol{k}_{7}\right)+v^{3} P\left(\boldsymbol{k}_{8}\right)\right) z \\
& +\left(v^{4} P\left(\boldsymbol{f}_{0}\right)+v^{2} P\left(\boldsymbol{f}_{+}\right)\right) z^{2},
\end{aligned}
$$

where $\boldsymbol{k}_{5}, \ldots, \boldsymbol{k}_{8}$ are tangles as shown in Figure 6. Since $\boldsymbol{k}_{5}, \boldsymbol{k}_{6}, \boldsymbol{k}_{7}, \boldsymbol{k}_{8}$ are the mirror images of $\boldsymbol{k}_{4}, \boldsymbol{k}_{3}, \boldsymbol{k}_{2}, \boldsymbol{k}_{1}$, respectively, by taking mirror images, (27) becomes

$$
\begin{aligned}
P\left(\boldsymbol{t}_{-}\right)= & v^{-8} P\left(\boldsymbol{e}_{+}\right)+\left(-v^{-7} P\left(\boldsymbol{k}_{4}\right)-v^{-5} P\left(\boldsymbol{k}_{3}\right)-v^{-5} P\left(\boldsymbol{k}_{2}\right)-v^{-3} P\left(\boldsymbol{k}_{1}\right)\right) z \\
& +\left(v^{-4} P\left(\boldsymbol{f}_{0}\right)+v^{-2} P\left(\boldsymbol{f}_{-}\right)\right) z^{2} .
\end{aligned}
$$

In fact, the HOMFLYPT polynomial of the mirror image $L$ ! of a link $L$ is obtained from that of $L$ by the formula

$$
P(L ! ; v, z)=P\left(L ;-v^{-1}, z\right) .
$$

Combining (26) and (28), we obtain (6).

\section{Two-cable links}

Let $L=K_{1} \cup \cdots \cup K_{n}$ be an oriented link with $n$ components and $N_{i}$ be a tubular neighborhood of $K_{i}$ such that $N_{1}, \ldots, N_{n}$ are disjoint. For integers $p_{1}, \ldots, p_{n}$, let $T\left(p_{i}\right)$ be a torus link of type $\left(2, p_{i}\right)$ on a solid torus $V_{i}$.

Let $\varphi_{i}: V_{i} \rightarrow N_{i}$ be a faithful homeomorphism, that is, the homeomorphism $\varphi_{i}$ sends the standard meridian-longitude system of $V_{i}$ to a standard meridian-longitude system of $N_{i}$. Then we call the link $\varphi_{1}\left(T\left(p_{1}\right)\right) \cup \cdots \cup \varphi_{n}\left(T\left(p_{n}\right)\right)$ the 2 -cable link about $L$ with framing $\boldsymbol{p}, \boldsymbol{p}=\left(p_{1}, \ldots, p_{n}\right)$, which we denote by $\widetilde{L} \boldsymbol{p}$ or $\widetilde{K}_{1}^{p_{1}} \cup \cdots \cup \widetilde{K}_{n}^{p_{n}}$; cf Rolfsen [25, Section 4D]. We assume that the strands in $\tilde{L}^{p}$ are oriented so that when they are stuck together to make the companion link $L$, their directions are parallel and agree with the orientation of $L$

We present a 2 -cable link $\widetilde{K}_{1}^{p_{1}} \cup \cdots \cup \widetilde{K}_{n}^{p_{n}}$ by drawing a diagram of the link $K_{1} \cup$ $\cdots \cup K_{n}$ together with the framings $p_{1}, \ldots, p_{n}$ near their respective components.

We describe this construction diagrammatically. Let $n, m$ be positive integers. In a diagram circles labeled $n, 0,-n, \infty, 1 / m,-1 / m$ stand for an $n$ tangle, a 0 tangle, a $-n$ tangle, an $\infty$ tangle, a $1 / m$ tangle, a $-1 / m$ tangle, respectively, as shown in Figure 7. 


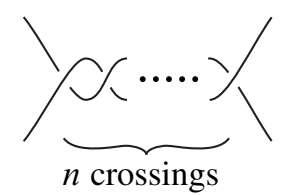

(a)

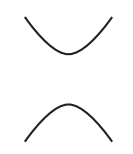

(b)

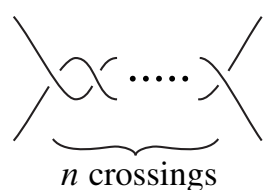

(c)

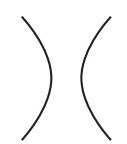

(d)

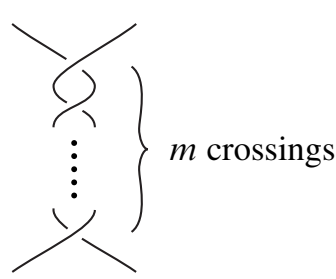

(e)

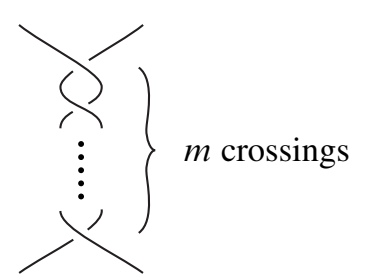

(f)

Figure 7: (a) $n$ tangle, (b) 0 tangle, (c) $-n$ tangle, (d) $\infty$ tangle, (e) $1 / m$ tangle, (f) $-1 / m$ tangle.

Figure 8 shows a diagram of the torus link of type $(2, p), T(p)$. Let $K$ be an oriented knot with diagram as shown in Figure 9(a), where $s$ is a 2-end tangle. Suppose that the writhe or algebraic crossing number of this diagram is $w$, that is, the number of positive crossings minus that of negative crossings. Then the 2 -cable link about $K$ with framing $p$ has a diagram as in Figure 9(b), where $m=2 w-p$ and the strands and crossings in $\boldsymbol{s}$ are transformed in the 4-end tangle diagram $\tilde{\boldsymbol{s}}$ as shown in Figure 9(c).

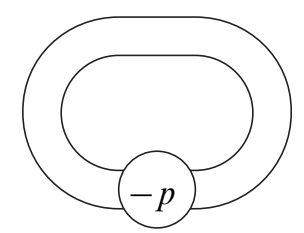

Figure 8: Torus link of type $(2, p), T(p)$.

Let $L=K_{1} \cup K_{2}$ be an oriented 2-component link with diagram as shown in Figure 10(a), where $t$ is a 4-end tangle and the lower strand belongs to $K_{1}$ and the upper one to $K_{2}$. Let $w_{i}$ be the writhe of $K_{i}$ in this diagram, $i=1,2$, that is, the number of positive self-crossings of $K_{i}$ minus that of negative self-crossings of $K_{i}$. Then the 2 -cable link about $L$ with framing $\left(p_{1}, p_{2}\right)$ has an diagram as in Figure 10(b), where 


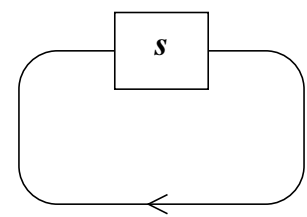

(a)

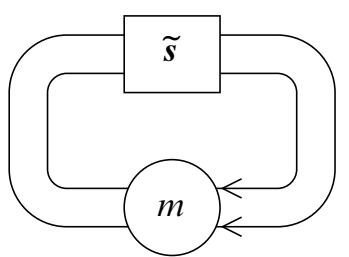

(b)

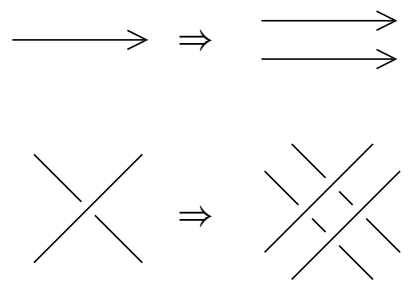

(c)

Figure 9

$m_{i}=2 w_{i}-p_{i}$ and the strands and crossings in $t$ are transformed as in the above. Note that the writhe of $L$ is $w_{1}+w_{2}+2 l$, where $l$ is the linking number of $K_{1}$ and $K_{2} ; l=\operatorname{lk}\left(K_{1}, K_{2}\right)$.

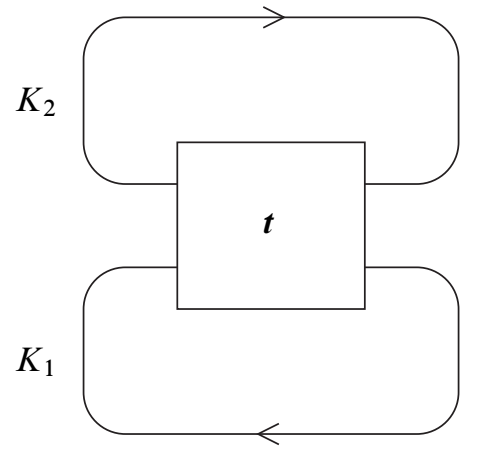

(a)

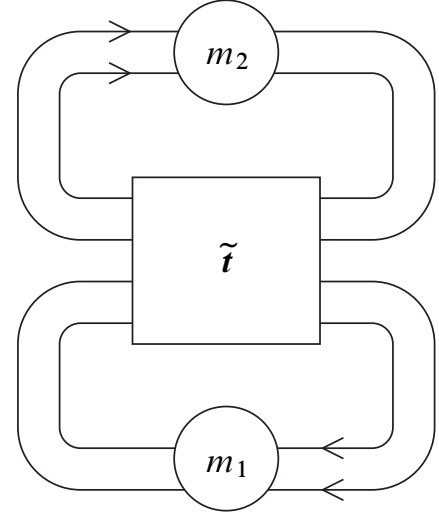

(b)

Figure 10

Now we give some properties of the HOMFLYPT polynomials of 2-cable links. Let $L=K \cup M$ be a link, where $K$ is a component and $M$ is a complement of $K$ in $L$. We consider the HOMFLYPT polynomial of its 2-cable link $\widetilde{L}^{p}=\widetilde{K}^{p} \cup \tilde{M}$, where $\tilde{M}$ is a 2-cable link of $M$.

Algebraic $8 \mathcal{G}$ Geometric Topology, Volume 7 (2007) 
For an integer $k$, we define a symmetric polynomial $\Phi_{k}(v, z) \in Z\left[v^{ \pm 1}, z^{ \pm 1}\right]$ as follows:

$$
\Phi_{k}(v, z)= \begin{cases}\frac{\varphi^{k}-\psi^{k}}{\varphi-\psi} & \text { if } k>0 \\ 0 & \text { if } k=0 \\ (-1)^{k-1} v^{2 k} \frac{\varphi^{-k}-\psi^{-k}}{\varphi-\psi}=(-1)^{k-1} v^{2 k} \Phi_{-k}(v, z) & \text { if } k<0\end{cases}
$$

where $\varphi, \psi \in Z\left[v^{ \pm 1}, z^{ \pm 1}\right]$ are defined by $\varphi \psi=-v^{2}$ and $\varphi+\psi=z$. Then we can prove the following by induction.

Proposition 3.1 For an integer $k$, we have

$$
P\left(\widetilde{L}^{k}\right)=\Phi_{k} P\left(\widetilde{L}^{1}\right)+v^{2} \Phi_{k-1} P\left(\tilde{L}^{0}\right) .
$$

Thus the HOMFLYPT polynomial of a 2 -cable link with framing $\left(k_{1}, \ldots, k_{n}\right), k_{i} \in \boldsymbol{Z}$, is obtained from those of the 2 -cable links with framings $\left(\epsilon_{1}, \ldots, \epsilon_{n}\right), \epsilon_{i}=0,1$.

Next we consider the HOMFLYPT polynomials of 2-cable links of a composite link. Let $L_{1}=J \cup M_{1}$ and $L_{2}=K \cup M_{2}$ be oriented links, where $J, K$ are components and $M_{1}, M_{2}$ are complements of $J, K$ in $L_{1}, L_{2}$, respectively, possibly empty. We construct a composite link by connecting the components $J$ and $K$; $L_{1} \# L_{2}=J \# K \cup M_{1} \cup M_{2}$. We put

$$
\begin{aligned}
\widetilde{L}_{1}^{j} & =\widetilde{J}^{j} \cup \tilde{M}_{1} ; \\
\tilde{L}_{2}^{k} & =\widetilde{K}^{k} \cup \tilde{M}_{2} ; \\
\widetilde{L}^{l} & =(\widetilde{J \# K})^{l} \cup \tilde{M}_{1} \cup \tilde{M}_{2},
\end{aligned}
$$

where $\tilde{M}_{1}, \tilde{M}_{2}$ are 2 -cable links about $M_{1}, M_{2}$, respectively. Then we have the following proposition.

Proposition 3.2 For integers $j, k, l$ with $l=j+k$, we have

$$
\begin{aligned}
\left(\mu^{2}-1\right) P\left(\tilde{L}^{l}\right)= & \mu\left(P\left(\tilde{L}_{1}^{j}\right) P\left(\tilde{L}_{2}^{k}\right)+P\left(\tilde{L}_{1}^{j-1}\right) P\left(\tilde{L}_{2}^{k+1}\right)\right) \\
& -\left(P\left(\tilde{L}_{1}^{j}\right) P\left(\tilde{L}_{2}^{k+1}\right)+P\left(\tilde{L}_{1}^{j-1}\right) P\left(\tilde{L}_{2}^{k}\right)\right)
\end{aligned}
$$

where $\mu=\left(v^{-1}-v\right) z^{-1}$ is the HOMFLYPT polynomial of the trivial 2-component link. 
Proof For the diagrams of $L_{1}, L_{2}, L_{1} \# L_{2}$ given as in Figure 11(a), where $\boldsymbol{s}$ and $\boldsymbol{t}$ are 2-end tangles, we may give the diagrams of their 2-cable links $\widetilde{L}_{1}^{j}, \widetilde{L}_{2}^{k}, \widetilde{L}^{l}$ as in Figure 11(b), where $\widetilde{\boldsymbol{s}}$ and $\tilde{\boldsymbol{t}}$ are 2-cable tangles about $\boldsymbol{s}$ and $\boldsymbol{t}$, respectively. The link $\widetilde{L}^{l}$ is isotopic to the link given in Figure 11(c), which is the sum of two 4-end tangles. By Lickorish and Millett [20, Proposition 12] the HOMFLYPT polynomial of the link given in Figure 11(c) is calculated from the four links $\widetilde{L}_{1}^{j}, \widetilde{L}_{1}^{j-1}, \widetilde{L}_{2}^{k}, \widetilde{L}_{2}^{k+1}$ as shown in Figure 11(d), and we obtain (35).

(a)
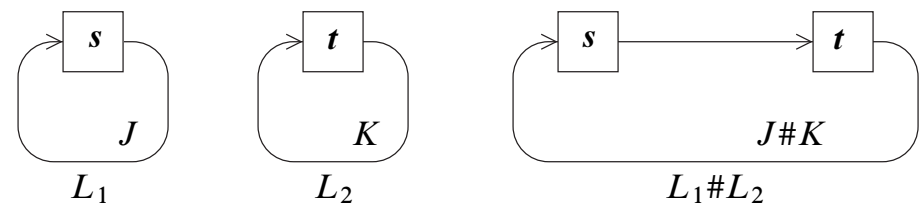

(b)
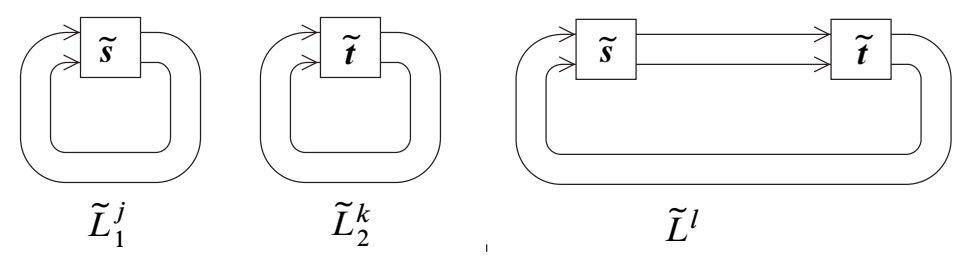

(c)

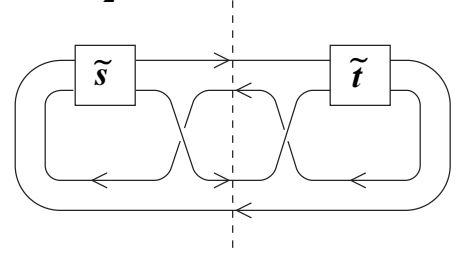

(d)
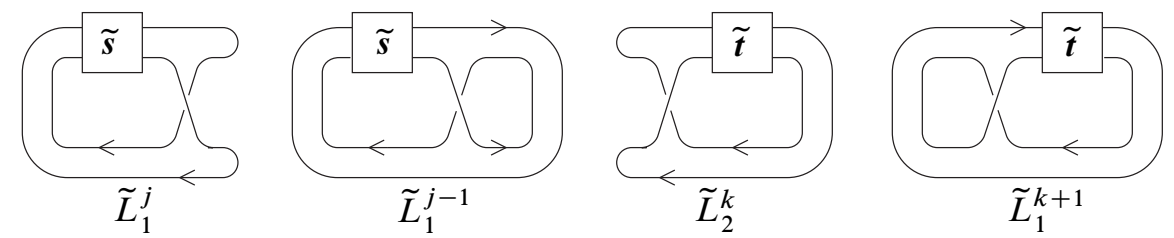

Figure 11

We apply Theorem 2.1 to 2 -cable links. Let $L_{+2}, L_{-2}, L_{0}, L_{\infty}$ be four oriented links that are identical except near one point where they are as in Figure 12.

We have two cases:

Case 1: The two strands of $L_{s}, s=+2,-2,0$, in Figure 12 belong to the same component, and those of $L_{\infty}$ belong to different components.

Case 2: The two strands of $L_{s}, s=+2,-2,0$, in Figure 12 belong to different components, and those of $L_{\infty}$ belong to the same component. 

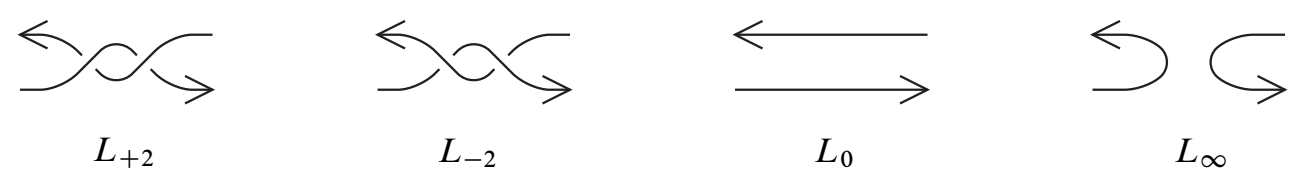

Figure 12

Case 1 We put

$$
\begin{aligned}
L_{s} & =K_{s} \cup M ; \\
L_{\infty} & =J_{1} \cup J_{2} \cup M,
\end{aligned}
$$

where $M$ is the common sublink of $L_{s}$ and $L_{\infty}$, possibly empty and $K_{s}, J_{1}, J_{2}$ are the visible components in Figure 12.

For integers $p, q$, we put their 2-cable links as follows.

$$
\begin{gathered}
\widetilde{L}_{s}^{p}=\widetilde{K}_{s}^{p} \cup \tilde{M} ; \\
\widetilde{L}_{\infty}^{(q, r)}=\widetilde{J}_{1}^{q} \cup \widetilde{J}_{2}^{r} \cup \tilde{M},
\end{gathered}
$$

where $\tilde{M}$ is a 2 -cable link about $M$. Then from Theorem 2.1, we have:

\section{Corollary 3.3}

$$
\begin{aligned}
& v^{-5} P\left(\widetilde{L}_{+2}^{p+2}\right)+v^{5} P\left(\widetilde{L}_{-2}^{p-2}\right)=v^{-3} P\left(\widetilde{L}_{0}^{p+2}\right)+v^{3} P\left(\widetilde{L}_{0}^{p-2}\right) \\
& \quad+\left(v^{-3} P\left(\widetilde{L}_{\infty}^{(q+1, r+1)}\right)+\left(v^{-1}+v\right) P\left(\widetilde{L}_{\infty}^{(q, r)}\right)+v^{3} P\left(\widetilde{L}_{\infty}^{(q-1, r-1)}\right)\right) z^{2}
\end{aligned}
$$

where $p=q+r+4 \operatorname{lk}\left(J_{1}, J_{2}\right)$.

Case 2 We put

$$
\begin{aligned}
L_{s} & =J_{s} \cup K_{s} \cup M ; \\
L_{\infty} & =H \cup M,
\end{aligned}
$$

where $M$ is the common sublink of $L_{s}$ and $L_{\infty}$, possibly empty and $J_{s}, K_{s}, H$ are the visible components in Figure 12.

For integers $p, q$, we put their 2-cable links as follows.

$$
\begin{aligned}
\tilde{L}_{s}^{(p, q)} & =\widetilde{J}_{s}^{p} \cup \tilde{K}_{s}^{q} \cup \tilde{M} ; \\
\widetilde{L}_{\infty}^{r} & =\tilde{H}^{r} \cup \tilde{M},
\end{aligned}
$$


where $\tilde{M}$ is a 2 -cable link about $M$. Then from Theorem 2.1, we have the following corollary.

\section{Corollary 3.4}

$$
\begin{aligned}
& v^{-5} P\left(\widetilde{L}_{+2}^{(p-1, q-1)}\right)+v^{5} P\left(\widetilde{L}_{-2}^{(p+1, q+1)}\right)=v^{-3} P\left(\widetilde{L}_{0}^{(p+1, q+1)}\right) \\
+ & v^{3} P\left(\widetilde{L}_{0}^{(p-1, q-1)}\right)+\left(v^{-3} P\left(\widetilde{L}_{\infty}^{r+2}\right)+\left(v^{-1}+v\right) P\left(\widetilde{L}_{\infty}^{r}\right)+v^{3} P\left(\widetilde{L}_{\infty}^{r-2}\right)\right) z^{2},
\end{aligned}
$$

where $r=p+q+4 \operatorname{lk}\left(J_{0}, K_{0}\right)$.

\section{The two-cable HOMFLYPT polynomials of the link $K\left[\beta ; R_{1}, R_{2}, \ldots, R_{n}\right]$}

In [8, Section 4], for a pure 3-braid $\beta$ and tangles $R_{1}, R_{2}, \ldots, R_{n-1}, R_{n}$, we defined a class of oriented links $K\left[\beta ; R_{1}, R_{2}, \ldots, R_{n-1}, R_{n}\right]$ as shown in Figure 13; if $n=0$, we interpret it as the 2 -bridge knot $K[\beta]$ as shown in Figure 13(c).

(a)

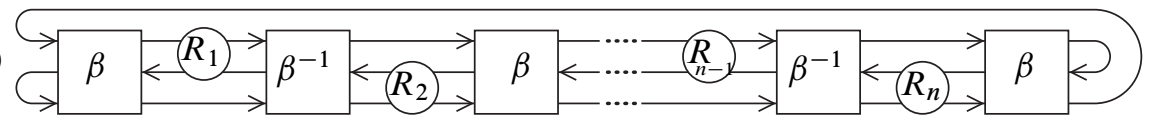

(b)

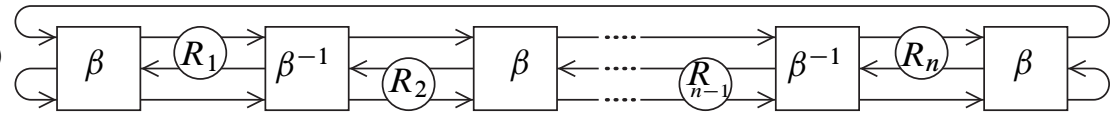

(c)

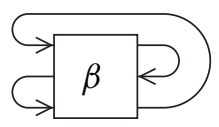

Figure 13: The link $K\left[\beta ; R_{1}, R_{2}, \ldots, R_{n-1}, R_{n}\right]$ with (a) $n$ even, (b) $n$ odd, (c) $n=0$.

We say that a 3-braid is strongly amphicheiral if it is of the form

$$
\left(\sigma_{2}^{q_{1}} \sigma_{1}^{q_{2}} \cdots\right)\left(\cdots \sigma_{2}^{-q_{2}} \sigma_{1}^{-q_{1}}\right)
$$

the closure of such a 3-braid is strongly amphicheiral in the sense of Van Buskirk [27]. Here $\sigma_{1}$ and $\sigma_{2}$ are elementary 3-braids as shown in Figure 14. Note that if a strongly 
amphicheiral 3-braid $\beta$ can be oriented as in Figure 13(c), then it is easy to see that $\beta$ is a pure braid.

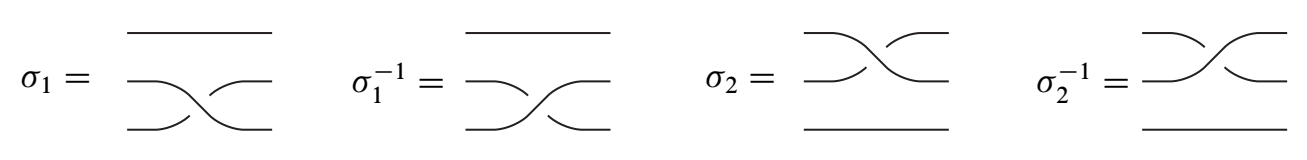

Figure 14: Elementary 3-braids.

For $r_{i} \in Z \cup\{\infty\}$, we denote by $K\left[\beta ; r_{1}, r_{2}, \ldots, r_{n}\right]$ the link $K\left[\beta ; R_{1}, R_{2}, \ldots, R_{n}\right]$ with each tangle $R_{i}$ the $r_{i}$ tangle. If $\beta$ is a strongly amphicheiral pure 3 -braid and each $r_{i}$ is an even integer, then $K\left[\beta ; r_{1}, r_{2}, \ldots, r_{n}\right]$ is a 2 -bridge knot or link (with 2 components) according as $n$ is even or odd. Note that a 2 -bridge link is interchangeable, that is, there is an isotopy of the 3-sphere which interchange the two components, and so we do not have to distinguish the components. We will prove the following theorem on 2 -cable links about the 2 -bridge links $K\left[\beta ; r_{1}, r_{2}, \ldots, r_{n}\right]$.

Theorem 4.1 If $\beta$ is a strongly amphicheiral pure 3 -braid and $r_{1}, r_{2}, \ldots, r_{n-1}, r_{n}$ are even integers, then

$$
P\left(\tilde{K}\left[\beta ; r_{1}, r_{2}, \ldots, r_{n-1}, r_{n}\right]^{\boldsymbol{p}}\right)=P\left(\tilde{K}\left[\beta ; r_{n}, r_{n-1}, \ldots, r_{2}, r_{1}\right] \boldsymbol{p}^{\boldsymbol{p}}\right)
$$

for any framing $\boldsymbol{p}$.

In order to prove this theorem, we need the following lemmas: Lemma 4.2 is a special case of [8, Lemma 4.2], and for Lemma 4.3, compare [8, Lemma 4.3].

Lemma 4.2 Suppose $\beta$ is a pure 3 -braid. If $r_{k}=0,1 \leq k \leq n$, then $K\left[\beta ; r_{1}, \ldots, r_{n}\right]$ is isotopic to:

$$
\begin{cases}\text { the trivial 2-component link } & \text { if } n=k=1 ; \\ K\left[\beta ; r_{3}, \ldots, r_{n}\right] & \text { if } n \geq 2, k=1 ; \\ K\left[\beta ; r_{1}, \ldots, r_{k-2}, r_{k-1}+r_{k+1}, r_{k+2}, \ldots, r_{n}\right] & \text { if } 2 \leq k \leq n-1 ; \\ K\left[\beta ; r_{1}, \ldots, r_{n-2}\right] & \text { if } n \geq 2, k=n,\end{cases}
$$

where both $K\left[\beta ; r_{3}, \ldots, r_{n}\right]$ and $K\left[\beta ; r_{1}, \ldots, r_{n-2}\right]$ with $n=2$ mean $K[\beta]$.

Lemma 4.3 Suppose that $\beta$ is a strongly amphicheiral pure 3-braid. If $r_{k}=\infty$, $1 \leq k \leq n$, then $K\left[\beta ; r_{1}, r_{2}, \ldots, r_{n}\right]$ is isotopic to the connected sum

$$
K\left[\beta ; r_{1}, r_{2}, \ldots, r_{k-1}\right] \# K\left[\beta ; r_{k+1}, r_{k+2}, \ldots, r_{n}\right],
$$

Algebraic $8 \mathcal{G}$ Geometric Topology, Volume 7 (2007) 
where both $K\left[\beta ; r_{1}, \ldots, r_{k-1}\right]$ with $k=1$ and $K\left[\beta ; r_{k+1}, \ldots, r_{n}\right]$ with $k=n$ mean $K[\beta]$.

Proof of Theorem 4.1 We prove by induction on $n$. The case $n=1$ is trivial. Let $m$ be a fixed integer with $m \geq 2$. Assuming that (47) holds for $n<m$, we will prove (47) with $n=m$.

Claim 1 If $r_{k}=0$ for some $k, 1 \leq k \leq m$, then (47) with $n=m$ holds for any even integers $r_{j}, j \neq k$, any strongly amphicheiral pure 3-braid $\beta$, and any framing $\boldsymbol{p}$.

Proof This follows from Lemma 4.2 and inductive hypothesis that (47) holds for $n<m$.

Claim 2 If $r_{k}=\infty$ for some $k, 1 \leq k \leq m$, then (47) with $n=m$ holds for any even integers $r_{j}, j \neq k$, any strongly amphicheiral pure 3-braid $\beta$, and any framing $\boldsymbol{p}$.

Proof There are four cases:

- Case 1. $m$ is even and $k$ is even.

- Case 2. $m$ is even and $k$ is odd.

- Case 3. $m$ is odd and $k$ is even.

- Case 4. $m$ is odd and $k$ is odd.

We prove only for Case 1 , since other cases are similar. We put $K_{1}=K\left[\beta ; r_{1}, \ldots, r_{m}\right]$, $K_{2}=K\left[\beta ; r_{m}, \ldots, r_{1}\right]$. By Lemma $4.3, K_{1}$ is isotopic to the connected sum of the 2-bridge link $K\left[\beta ; r_{1}, \ldots, r_{k-1}\right]$ and the 2 -bridge knot $K\left[\beta ; r_{k+1}, \ldots, r_{m}\right]$, which we denote by $L_{1}$ and $J_{1}$, respectively, and $K_{2}$ is isotopic to the connected sum of the 2-bridge link $K\left[\beta ; r_{k-1}, \ldots, r_{1}\right]$ and the 2 -bridge knot $K\left[\beta ; r_{m}, \ldots, r_{k+1}\right]$, which we denote by $L_{2}$ and $J_{2}$, respectively. Thus $K_{s}=L_{s} \# J_{s}, s=1,2$. By Proposition 3.2 , we have

$$
\begin{aligned}
\left(\mu^{2}-1\right) P\left(\tilde{K}_{s}^{k}\right)= & \mu\left(P\left(\widetilde{L}_{s}^{\left(l, l^{\prime}\right)}\right) P\left(\widetilde{J}_{s}^{j}\right)+P\left(\widetilde{L}_{s}^{\left(l-1, l^{\prime}\right)}\right) P\left(\widetilde{J}_{s}^{j+1}\right)\right) \\
& -\left(P\left(\widetilde{L}_{s}^{\left(l, l^{\prime}\right)}\right) P\left(\widetilde{J}_{s}^{j+1}\right)+P\left(\widetilde{L}_{s}^{\left(l-1, l^{\prime}\right)}\right) P\left(\widetilde{J}_{s}^{j}\right)\right)
\end{aligned}
$$

where $k=l+j$. By the inductive hypothesis, $P\left(\widetilde{L}_{1}^{\left(l, l^{\prime}\right)}\right)=P\left(\tilde{L}_{2}^{\left(l, l^{\prime}\right)}\right)$ for any integers $l, l^{\prime}$, and $P\left(\widetilde{J}_{s}^{j}\right)=P\left(\widetilde{J}_{s}^{j}\right)$ for any integer $j$. Thus we have $P\left(\widetilde{K}_{1}^{k}\right)=P\left(\widetilde{K}_{2}^{k}\right)$, completing the proof. 
Claim 3 Let $1 \leq k \leq m$. Suppose that (47) with $n=m$ and $r_{k}=r_{k+1}=\cdots=r_{m}=2$ holds for any even integers $r_{1}, \ldots, r_{k-1}$, any strongly amphicheiral pure 3 -braid $\beta$, and any framing $\boldsymbol{p}$. Then (47) with $n=m$ and $r_{k+1}=\cdots=r_{m}=2$ holds for any even integers $r_{1}, \ldots, r_{k-1}, r_{k}$, any strongly amphicheiral pure 3 -braid $\beta$, and any framing p .

Proof We prove by induction on $r_{k}$. There are four cases:

- Case 1. $m$ is even and $k$ is even.

- Case 2. $m$ is even and $k$ is odd.

- Case 3. $m$ is odd and $k$ is even.

- Case 4. $m$ is odd and $k$ is odd.

We prove only for Case 1, since other cases are similar. In this case both of

$$
\begin{aligned}
& K[\beta ; r_{1}, \ldots, r_{k-1}, r, \underbrace{2, \ldots, 2}_{m-k}], \\
& K[\beta ; \underbrace{2, \ldots, 2}_{m-k}, r, r_{k-1}, \ldots, r_{1}]
\end{aligned}
$$

are 2-bridge knots, so we denote their 2-cable links with framing $p$ by $\widetilde{H}_{r}^{p}, \widetilde{J}_{r}^{p}$, respectively.

We show

$$
P\left(\widetilde{H}_{r}^{p}\right)=P\left(\widetilde{J}_{r}^{p}\right)
$$

for any integer $p$ by induction on an even integer $r$. The case $r=0$ follows from Claim 1. The case $r=2$ is the condition of Claim 3. By (40) in Corollary 3.3, we have

$$
\begin{aligned}
& v^{-5} P\left(\tilde{H}_{r+2}^{q+2}\right)-v^{-3} P\left(\tilde{H}_{r}^{q+2}\right)-v^{3} P\left(\tilde{H}_{r}^{q-2}\right)+v^{5} P\left(\tilde{H}_{r-2}^{q-2}\right) \\
& =\left(v^{-3} P\left(\widetilde{H}_{\infty}^{(i+1, j+1)}\right)+\left(v^{-1}+v\right) P\left(\widetilde{H}_{\infty}^{(i, j)}\right)+v^{3} P\left(\widetilde{H}_{\infty}^{(i-1, j-1)}\right)\right) z^{2} \\
& v^{-5} P\left(\widetilde{J}_{r+2}^{q+2}\right)-v^{-3} P\left(\widetilde{J}_{r}^{q+2}\right)-v^{3} P\left(\widetilde{J}_{r}^{q-2}\right)+v^{5} P\left(\widetilde{J}_{r-2}^{q-2}\right) \\
& =\left(v^{-3} P\left(\widetilde{J}_{\infty}^{(i+1, j+1)}\right)+\left(v^{-1}+v\right) P\left(\widetilde{J}_{\infty}^{(i, j)}\right)+v^{3} P\left(\widetilde{J}_{\infty}^{(i-1, j-1)}\right)\right) z^{2},
\end{aligned}
$$

where $q=i+j+2\left(r_{1}+r_{3}+\cdots+r_{k-3}+r_{k-1}\right)$. By Claim 2, the right hand side of (52) and that of (53) are equal. Thus if (51) holds for $r=l, l+2$, then (51) holds for $r=l-2, l+4$. This completes the proof of (51). 
Since (47) with $n=m$ and $r_{1}=r_{2}=\cdots=r_{m}=2$ is trivial, by induction on $k$ Claim 3 completes the proof of (47) with $n=m$ and any even integers $r_{1}, r_{2}, \ldots, r_{m}$. This completes the proof of Theorem 4.1.

For integers $r_{i}(\neq 0)$, we denote by $K\left[\beta ; 1 / r_{1}, 1 / r_{2}\right]$ the link $K\left[\beta ; R_{1}, R_{2}\right]$ with $R_{i}$ the $1 / r_{i}$ tangle. If each $r_{i}$ is even, then $K\left[\beta ; 1 / r_{1}, 1 / r_{2}\right]$ is a knot. We can prove the following in a similar way to Theorem 4.1.

Theorem 4.4 If $\beta$ is a strongly amphicheiral pure 3-braid and $r_{1}, r_{2}$ are even integers, then the 2-cable links with the same framing of the knots

$$
K\left[\beta ; 1 / r_{1}, 1 / r_{2}\right], \quad K\left[\beta ; 1 / r_{2}, 1 / r_{1}\right]
$$

share the same HOMFLYPT polynomial.

Corollary 4.5 The pair of knots (54) share the same HOMFLYPT, Kauffman, LG polynomials and their 2-cable links share the same HOMFLYPT polynomials.

Proof For the HOMFLYPT and Kauffman polynomials, we can prove in a similar way to Kauffman [13, Propositions 1 and 3], respectively. For the LG polynomial, this follows from [8, Theorem 4.1].

Example 4.6 According to Corollary 4.5, it is not easy to distinguish the knot pairs (54). For a given pair, we can distinguish by applying the computer program SnapPea of Jeffrey R Weeks. For example, the hyperbolic volumes of

$$
K\left[\sigma_{2}^{2} \sigma_{1}^{-2} ; 1 / 2,-1 / 2\right], \quad K\left[\sigma_{2}^{2} \sigma_{1}^{-2} ;-1 / 2,1 / 2\right]
$$

are 18.0277914698 and 18.120528841550 , respectively.

Remark 4.7 For a pure 3-braid $\beta$ and tangles $R_{1}, R_{2}, \ldots, R_{n}$, with $n$ even, let $L\left[\beta ; R_{1}, R_{2}, \ldots, R_{n}\right]$ be an oriented link as shown in Figure 15 [8, Figure 10]; for an oriented knot $J$ and an integer $p$, let $\Sigma\left[\beta ; R_{1}, R_{2}, \ldots, R_{n} ; J, p\right]$ be the satellite link with companion $J$, pattern $L\left[\beta ; R_{1}, R_{2}, \ldots, R_{n}\right]$, and twisting number $p$ as defined in [8, page 282]. If each tangle $R_{i}$ is an $r_{i}$ tangle, where $r_{i}$ is an even integer, we denote these links by $L\left[\beta ; r_{1}, r_{2}, \ldots, r_{n}\right]$ and $\Sigma\left[\beta ; r_{1}, r_{2}, \ldots, r_{n} ; J, p\right]$, which are 3 -component link. We can prove that the 2 -cable links of $L\left[\beta ; r_{1}, r_{2}, \ldots, r_{n}\right]$ and $L\left[\beta ; r_{n}, \ldots, r_{2}, r_{1}\right]\left(\right.$ resp. $\Sigma\left[\beta ; r_{1}, r_{2}, \ldots, r_{n} ; J, p\right]$ and $\left.\Sigma\left[\beta ; r_{n}, \ldots, r_{2}, r_{1} ; J, p\right]\right)$ with the same framing share the same HOMFLYPT polynomial in a similar way to $[8$, Theorem 5.1] and Theorem 4.1. 


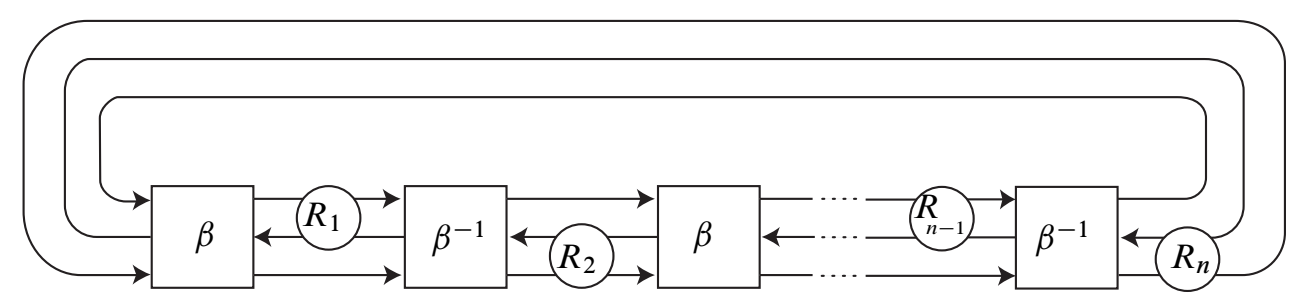

Figure 15: The link $L\left[\beta ; r_{1}, r_{2}, \ldots, r_{n}\right]$.

\section{The two-cable HOMFLYPT polynomials of 2-bridge links}

In this section, we show the following theorems using Theorem 4.1. See Conway [1] and Lickorish and Millett [20] for the skein equivalence; if two links are skein equivalent, then they share the same HOMFLYPT polynomial.

Theorem 5.1 For any positive integer $N$, there exist $2^{N}$, mutually distinct, amphicheiral, fibered 2-bridge knots, which are skein equivalent, share the same Kauffman and $L G$ polynomials, and whose 2-cable links with the same framing share the same HOMFLYPT polynomial.

Theorem 5.2 For any positive integer $N$, there exist $2^{N}$, mutually distinct, amphicheiral, fibered 2-bridge links, which are skein equivalent, share the same Kauffman, 2-variable Alexander, LG polynomials, and whose 2-cable links with the same framing share the same HOMFLYPT polynomial.

For a 3-braid $\alpha$ and integers $p_{1}, p_{2}, \ldots, p_{n-1}, p_{n}$, we define 3 -braids as follows:

$$
\begin{gathered}
\alpha\left\langle p_{1}\right\rangle=\alpha\left(p_{1},-p_{1}\right)=\alpha \boldsymbol{s}_{2}^{p_{1}} \alpha^{-1} s_{1}^{-p_{1}} \alpha ; \\
\alpha\left\langle p_{1}, p_{2}, \ldots, p_{n-1}, p_{n}\right\rangle=\alpha\left\langle p_{1}, p_{2}, \ldots, p_{n-1}\right\rangle\left\langle p_{n}\right\rangle .
\end{gathered}
$$

Then,

$$
\alpha\left\langle p_{1}, p_{2}, \ldots, p_{n-1}, p_{n}\right\rangle=\alpha\left\langle p_{1}, p_{2}, \ldots, p_{i-1}\right\rangle\left\langle p_{i}, \ldots, p_{n}\right\rangle,
$$

where $2 \leq i \leq n$, and

$$
\alpha\left\langle p_{1}, p_{2}, \ldots, p_{n}\right\rangle=\alpha\left(q_{1}, q_{2}, \ldots, q_{m-1}, q_{m}\right),
$$

where $m=3^{n}-1$ and $p_{i}=\epsilon_{j} q_{j}$ with $j \equiv 0\left(\bmod 3^{i-1}\right), j \not \equiv 0\left(\bmod 3^{i}\right)$, and

$$
\epsilon_{j}=\left\{\begin{array}{lll}
1 & \text { if } j / 3^{i-1} \equiv 1 \quad(\bmod 3) ; \\
-1 & \text { if } j / 3^{i-1} \equiv 2 \quad(\bmod 3)
\end{array}\right.
$$


cf [13, page 285]. Using Theorem 4.1, we can show the following lemma in a similar way to the proof of [8, Lemma 6.3].

Lemma 5.3 Suppose $\beta$ is a strongly amphicheiral pure 3-braid and $b_{1}, b_{2}, \ldots, b_{n}$ are integers. Then for any framing $\boldsymbol{p}$, we have

$$
P\left(\tilde{K}\left[\beta\left\langle 2 b_{1}, 2 b_{2}, \ldots, 2 b_{n}\right\rangle\right]^{\boldsymbol{p}}\right)=P\left(\tilde{K}\left[\beta\left\langle-2 b_{1},-2 b_{2}, \ldots,-2 b_{n}\right\rangle\right] \boldsymbol{p}\right) .
$$

For a nontrivial pure strongly amphicheiral 3 -braid $\beta$, we define the following sets consisting of $2^{N} 2$-bridge knots and links:

$$
\begin{aligned}
& \mathcal{K}_{\beta, N}=\left\{K\left[\beta\left\langle 2 \epsilon_{1}, 2 \epsilon_{2}, \ldots, 2 \epsilon_{N}\right\rangle\right] \mid \epsilon_{i}= \pm 1\right\} ; \\
& \mathcal{L}_{\beta, N}=\left\{K\left[\beta\left\langle 2 \epsilon_{1}, 2 \epsilon_{2}, \ldots, 2 \epsilon_{N}\right\rangle(2)\right] \mid \epsilon_{i}= \pm 1\right\} .
\end{aligned}
$$

Proof of Theorem 5.1 In [8], we have shown that the $2^{N}$ knots in $\mathcal{K}_{\beta, N}$ are mutually distinct, amphicheiral, fibered 2-bridge knots, which are skein equivalent, share the same Kauffman and LG polynomials. We then show that their 2-cable links share the same HOMFLYPT polynomial. In fact, for each $i, 1 \leq i \leq N$, we can show the following in the same way as [8, Equation (40)] using (56) and Lemma 5.3.

$$
\begin{aligned}
P\left(\tilde { K } \left[\beta \left\langle2 \epsilon_{1}, 2 \epsilon_{2}, \ldots,\right.\right.\right. & \left.\left.\left.2 \epsilon_{i-1}, 2 \epsilon_{i}, 2 \epsilon_{i+1}, \ldots, 2 \epsilon_{N}\right\rangle\right] \boldsymbol{p}\right) \\
= & P\left(\tilde{K}\left[\beta\left\langle 2 \epsilon_{1}, 2 \epsilon_{2}, \ldots, 2 \epsilon_{i-1},-2 \epsilon_{i}, 2 \epsilon_{i+1}, \ldots, 2 \epsilon_{N}\right\rangle\right] \boldsymbol{p}\right) .
\end{aligned}
$$

This completes the proof.

Proof of Theorem 5.2 The 2-bridge links in the above set $\mathcal{L}_{\beta, N}$ are the desired ones.

Example 5.4 According to [8, page 286], there are 11 pairs of 2-bridge knots and one pair of 2-bridge links through 20 crossings sharing the same HOMFLYPT, Kauffman, and LG polynomials; for 2-bridge knots,

$$
\begin{aligned}
& \left\{K\left[\sigma_{2}^{2} \sigma_{1}^{-2} ; p, q\right], K\left[\sigma_{2}^{2} \sigma_{1}^{-2} ; q, p\right]\right\}, \\
& \left\{K\left[\boldsymbol{s}_{2}^{2} \boldsymbol{s}_{1}^{2} \boldsymbol{s}_{2}^{-2} \boldsymbol{s}_{1}^{-2} ; 2,-2\right], K\left[\boldsymbol{s}_{2}^{2} \boldsymbol{s}_{1}^{2} \boldsymbol{s}_{2}^{-2} \boldsymbol{s}_{1}^{-2} ;-2,2\right]\right\},
\end{aligned}
$$

where $(p, q)=(2,-2),(4,2),(4,-2),(6,2),(6,-2),(4,-4),(8,2),(8,-2)$, $(6,4),(6,-4)$; and for $2-$ bridge links

$$
\left\{K\left[s_{2}^{2} s_{1}^{-2} ; 2,2,-2\right], K\left[s_{2}^{2} s_{1}^{-2} ;-2,2,2\right]\right\} .
$$

By Theorem 4.1, their 2-cable links also share the same HOMFLYPT polynomials. 
The simplest pair is

$$
\left\{K\left[\sigma_{2}^{2} \sigma_{1}^{-2} ; 2,-2\right], K\left[\sigma_{2}^{2} \sigma_{1}^{-2} ;-2,2\right]\right\},
$$

which are

$$
\{C(2,1,1,1,2,2,1,1,1,2), C(2,2,1,1,1,1,1,1,2,2)\}
$$

in Conway's presentation for 2-bridge knots, and thus have 14 crossings.

\section{References}

[1] J H Conway, An enumeration of knots and links, and some of their algebraic properties, from: "Computational Problems in Abstract Algebra (Proc. Conf., Oxford, 1967)", Pergamon, Oxford (1970) 329-358 MR0258014

[2] D De Wit, Automatic evaluation of the Links-Gould invariant for all prime knots of up to 10 crossings, J. Knot Theory Ramifications 9 (2000) 311-339 MR1753798

[3] D De Wit, J Links, Where the Links-Gould invariant first fails to distinguish nonmutant prime knots arXiv:math.GT/0501224v2

[4] D De Wit, J R Links, L H Kauffman, On the Links-Gould invariant of links, J. Knot Theory Ramifications 8 (1999) 165-199 MR1687549

[5] P Freyd, D Yetter, J Hoste, W B R Lickorish, K Millett, A Ocneanu, A new polynomial invariant of knots and links, Bull. Amer. Math. Soc. (N.S.) 12 (1985) 239-246 MR776477

[6] J Hoste, M Thistlethwaite, J Weeks, The first 1,701,936 knots, Math. Intelligencer 20 (1998) 33-48 MR1646740

[7] A Ishii, Algebraic links and skein relations of the Links-Gould invariant, Proc. Amer. Math. Soc. 132 (2004) 3741-3749 MR2084099

[8] A Ishii, T Kanenobu, Different links with the same Links-Gould invariant, Osaka J. Math. 42 (2005) 273-290 MR2147736

[9] V F R Jones, Hecke algebra representations of braid groups and link polynomials, Ann. of Math. (2) 126 (1987) 335-388 MR908150

[10] T Kanenobu, Infinitely many knots with the same polynomial invariant, Proc. Amer. Math. Soc. 97 (1986) 158-162 MR831406

[11] T Kanenobu, Examples on polynomial invariants of knots and links, Math. Ann. 275 (1986) 555-572 MR859330

[12] T Kanenobu, Examples on polynomial invariants of knots and links. II, Osaka J. Math. 26 (1989) 465-482 MR1021426 
[13] T Kanenobu, Kauffman polynomials for 2-bridge knots and links, Yokohama Math. J. 38 (1991) 145-154 MR1105072

[14] T Kanenobu, Genus and Kauffman polynomial of a 2-bridge knot, Osaka J. Math. 29 (1992) 635-651 MR1181126

[15] T Kanenobu, T Sumi, Polynomial invariants of 2-bridge links through 20 crossings, from: "Aspects of low-dimensional manifolds", Adv. Stud. Pure Math. 20, Kinokuniya, Tokyo (1992) 125-145 MR1208310

[16] T Kanenobu, T Sumi, Polynomial invariants of 2-bridge knots through 22 crossings, Math. Comp. 60 (1993) 771-778, S17-S28 MR1176711

[17] L H Kauffman, On Knots, volume 115 of Ann. of Math. Studies, Princeton University Press, Princeton (1987)

[18] L H Kauffman, An invariant of regular isotopy, Trans. Amer. Math. Soc. 318 (1990) 417-471 MR958895

[19] W B R Lickorish, A S Lipson, Polynomials of 2-cable-like links, Proc. Amer. Math. Soc. 100 (1987) 355-361 MR884479

[20] W B R Lickorish, K C Millett, A polynomial invariant of oriented links, Topology 26 (1987) 107-141 MR880512

[21] J R Links, M D Gould, Two variable link polynomials from quantum supergroups, Lett. Math. Phys. 26 (1992) 187-198 MR1199742

[22] H R Morton, H B Short, The 2-variable polynomial of cable knots, Math. Proc. Cambridge Philos. Soc. 101 (1987) 267-278 MR870598

[23] J H Przytycki, Equivalence of cables of mutants of knots, Canad. J. Math. 41 (1989) 250-273 MR1001611

[24] J H Przytycki, P Traczyk, Invariants of links of Conway type, Kobe J. Math. 4 (1988) 115-139 MR945888

[25] D Rolfsen, Knots and links, Publish or Perish, Berkeley, CA (1976) MR0515288

[26] A Stoimenow, On cabled knots and Vassiliev invariants (not) contained in knot polynomials, Canad. J. Math. 59 (2007) 418-448 MR2310624

[27] J M Van Buskirk, A class of negative-amphicheiral knots and their Alexander polynomials, Rocky Mountain J. Math. 13 (1983) 413-422 MR715764

[28] S Yamada, On the two variable Jones polynomial of satellite links, from: "Topology and computer science (Atami, 1986)", Kinokuniya, Tokyo (1987) 295-300 MR1112599

Department of Mathematics, Osaka City University, Sugimoto, Sumiyoshi-ku, Osaka 558-8585, Japan

kanenobu@sci.osaka-cu.ac.jp

Received: 27 December 2005

Algebraic $6 \mathcal{G}$ Geometric Topology, Volume 7 (2007) 